\title{
Oscillations of the superconducting temperature induced by quantum well states in thin metallic films: Numerical solution of the Bogoliubov-de Gennes equations
}

\author{
A. A. Shanenko, ${ }^{1,2}$ M. D. Croitoru, ${ }^{3}$ and F. M. Peeters ${ }^{1}$ \\ ${ }^{1}$ TGM, Departement Fysica, Universiteit Antwerpen, Groenenborgerlaan 171, B-2020 Antwerpen, Belgium \\ ${ }^{2}$ Bogoliubov Laboratory of Theoretical Physics, Joint Institute for Nuclear Research, 141980 Dubna, Russia \\ ${ }^{3}$ EMAT, Departement Fysica, Universiteit Antwerpen, Groenenborgerlaan 171, B-2020 Antwerpen, Belgium
}

(Received 21 August 2006; published 16 January 2007)

\begin{abstract}
Quantum confinement of electrons in a high-quality metallic nanofilm results in the formation of quantumwell states. The band of single-electron states is split into a series of subbands moving in energy with changing film thickness. When the bottom of such a subband passes through the Fermi surface, a shape resonance appears leading to oscillations of the critical temperature $T_{c}$ as a function of film thickness. We present a quantitative description of recent experimental data on the film-thickness dependence of $T_{c}$ in $\mathrm{Pb}$ nanofilms with atomic-scale uniformity in thickness [Science 306, 1915 (2004)] through a numerical solution of the Bogoliubov-de Gennes equations.
\end{abstract}

DOI: 10.1103/PhysRevB.75.014519

PACS number(s): 74.78.Na

\section{INTRODUCTION}

When the characteristic size of a superconductor is of the order of the bulk Fermi wavelength $\lambda_{F}$ (for most metals, $\left.\lambda_{F} \sim 1 \mathrm{~nm}\right)$, quantum confinement of electrons plays a major role. For high-quality metallic films quantization of the electron motion in the direction normal to the film results in the formation of so-called quantum-well states (QWS), and the band structure of the single-electron states is split in a series of two-dimensional (2D) parabolic subbands. QWS move down in energy with increasing the film thickness. When a QWS passes through the Fermi surface, a new subband (related to this QWS) comes into play. If the film thickness is small enough, this passage is accompanied by a significant change of the density of single-electron states near the Fermi level. Consequently, the electronic properties of a thin metallic film will be greatly modulated by varying its thickness.

In agreement with these expectations, photoelectron spectroscopy has demonstrated clear signatures of the formation of such QWS's in thin crystalline metallic films. ${ }^{1}$ In addition, recent experimental studies have shown that the surface energy $^{2}$ and thermal stability ${ }^{3}$ strongly vary with changing film thickness. The variations were found to follow a damped oscillatory curve of Friedel-like form. ${ }^{2,3}$ Very recently, oscillations of the superconducting temperature $T_{c}$ and perpendicular upper critical magnetic field $H_{c 2 \perp}$ were also reported $^{4,5}$ for $\mathrm{Pb}$ nanofilms with atomic-scale uniformity in thickness. Contrary to previous results on the thickness dependence of $T_{c}$ in Sn (Ref. 6) and $\mathrm{Pb}$ (Ref. 7) films, this is a solid demonstration that the observed variations are correlated with the passages of QWS through the Fermi surface. Motivated by these experimental findings, we present a detailed theoretical investigation of the $T_{c}$ oscillations in various metallic nanofilms. Earlier works on this subject ${ }^{8}$ were limited to a simplified multiband BCS model and did not investigate how the $T_{c}$ oscillations are dependent on the relevant parameters. Furthermore, those works were pure theoretical and no comparison with experiment was given. Here we present a microscopic study which is based on the Bogoliubov-de Gennes (BdG) equations, ${ }^{9}$ where we include the important QWS and give a detailed comparison with recent experiments.

This paper is organized as follows. In Sec. II we outline the formalism of the BdG equations and apply it to nanofilms in the parabolic band (PB) approximation. Section III presents numerical results of the BdG equations solved in a self-consistent manner for various metallic nanofilms. We discuss shape superconducting resonances resulting in quantum oscillations of the critical temperature and the effect of such resonances on the superconducting order parameter. In Sec. IV we investigate how the $T_{c}$ oscillations are dependent on the Fermi level. It is shown that experimental results for the period of the oscillations are not reproduced by the BdG equations when using the PB approximation. Strictly speaking, the actual band structure in the presence of quantum confinement is needed to recover the right periodicity of the $T_{c}$ oscillations. Therefore, we introduce an effective Fermi level which is able to provide the correct period of the quantum oscillations within the PB approximation. In Sec. V we show that the experimental data for the critical temperature in $\mathrm{Pb}(111)$ nanofilms fabricated on a silicon substrate can be well described by our theoretical results if we incorporate also the interface effect on the electron-phonon coupling.

\section{THE BOGOLIUBOV-DE GENNES EQUATIONS FOR A FILM}

In the presence of quantum confinement translational invariance is broken and the superconducting order parameter depends on the position $\Delta=\Delta(\mathbf{r})$. It is well known that the Bogoliubov-de Gennes (BdG) equations ${ }^{9}$ are a powerful tool for investigating the superconducting properties in spatially inhomogeneous systems. These equations are written as

$$
\left(\begin{array}{cc}
H_{e} & \Delta(\mathbf{r}) \\
\Delta^{*}(\mathbf{r}) & -H_{e}^{*}
\end{array}\right)\left(\begin{array}{c}
u_{i}(\mathbf{r}) \\
v_{i}(\mathbf{r})
\end{array}\right)=E_{i}\left(\begin{array}{c}
u_{i}(\mathbf{r}) \\
v_{i}(\mathbf{r})
\end{array}\right),
$$

where for zero magnetic field the single-electron Hamiltonian $H_{e}$ is given by 


$$
H_{e}=-\frac{\hbar^{2}}{2 m} \nabla^{2}+U(\mathbf{r})-\mu
$$

with $\mu$ the chemical potential and $m$ the electron band mass set to the free electron mass here. To study quantum-size effects, we consider a crystalline nanofilm that is in the clean limit in the transverse direction $\left(L_{z} \leqslant l_{e}\right.$, where $l_{e}$ is the meanfree path and $L_{z}$ is film thickness). The periodic lattice potential is taken into consideration by introducing the electron band mass, i.e., the parabolic band (PB) approximation. We limit ourselves to zero applied magnetic field and consequently the order parameter can be chosen as a real quantity $\Delta(\mathbf{r})=\Delta^{*}(\mathbf{r})$. The normal and anomalous mean-field potentials are related to the eigenfunctions $u_{i}(\mathbf{r})$ and $v_{i}(\mathbf{r})$ through

$$
\begin{gathered}
U(\mathbf{r})=-g \sum_{i}\left[\left|u_{i}(\mathbf{r})\right|^{2} f_{i}+\left|v_{i}(\mathbf{r})\right|^{2}\left(1-f_{i}\right)\right], \\
\Delta(\mathbf{r})=g \sum_{i} u_{i}(\mathbf{r}) v_{i}^{*}(\mathbf{r})\left(1-2 f_{i}\right),
\end{gathered}
$$

with $g$ the electron-phonon coupling and $f_{i}=f\left(E_{i}\right)$ the Fermi function. The sum in Eq. (3a) is over all positive energies $E_{i}$. The same summation range applies in Eq. (3b) but in addition it is limited to the Debye window of the single-electron energies $\left|\xi_{i}\right|<\hbar \omega_{D}$, where $\omega_{D}$ is the Debye frequency and $\xi_{i}$ is given by

$$
\begin{aligned}
\xi_{i}= & \int d^{3} r\left[u_{i}^{*}(\mathbf{r})\left(-\frac{\hbar^{2}}{2 m} \nabla^{2}+U(\mathbf{r})-\mu\right) u_{i}(\mathbf{r})\right. \\
& \left.+v_{i}^{*}(\mathbf{r})\left(-\frac{\hbar^{2}}{2 m} \nabla^{2}+U(\mathbf{r})-\mu\right) v_{i}(\mathbf{r})\right] .
\end{aligned}
$$

Without this additional limitation the sum in Eq. (3b) is divergent due to the $\delta$-function approximation for the electronelectron interaction. ${ }^{9}$ The BdG equations should be solved together with the self-consistency relations (3a) and (3b) for a given chemical potential $\mu$ which is determined by

$$
n_{e}=\frac{2}{V} \int d^{3} r \sum_{i}\left[\left|u_{i}(\mathbf{r})\right|^{2} f_{i}+\left|v_{i}(\mathbf{r})\right|^{2}\left(1-f_{i}\right)\right],
$$

where $n_{e}$ is the mean electron density and $V=L_{x} L_{y} L_{z}$ is the system volume $\left(L_{z} \ll L_{x}=L_{y}\right)$. The sum in Eq. (5) is over all the states with positive $E_{i}$.

We use periodic boundary conditions in the plane of the film, i.e., for the $x$ and $y$ directions. In this case $\Delta(\mathbf{r})=\Delta(z)$ and $U(\mathbf{r})=U(z)$, and we may write

$$
\begin{aligned}
& u_{k_{x} k_{y} j}(\mathbf{r})=\frac{e^{i k_{x} x}}{\sqrt{L_{x}}} \frac{e^{i k_{y} y}}{\sqrt{L_{y}}} \widetilde{u}_{k_{x} k_{y} j}(z), \\
& v_{k_{x} k_{y} j}(\mathbf{r})=\frac{e^{i k_{x} x}}{\sqrt{L_{x}}} \frac{e^{i k_{y} y}}{\sqrt{L_{y}}} \widetilde{v}_{k_{x} k_{y} j}(z),
\end{aligned}
$$

where $i=\left\{k_{x}, k_{y}, j\right\}$ with $k_{x}\left(k_{y}\right)$ the free electron wave vector in $x(y)$ direction and $j$ the quantum number related to the electron motion in the $z$ direction normal to the film. The normalization condition

$$
\int d z\left[\left|\widetilde{u}_{i}(z)\right|^{2}+\left|\widetilde{v}_{i}(z)\right|^{2}\right]=1
$$

is implied in Eqs. (6a) and (6b) (see, for instance, Ref. 9). Due to the electron confinement in the $z$ direction we have

$$
\widetilde{u}_{i}(0)=\widetilde{u}_{i}\left(L_{z}\right)=0, \quad \widetilde{v}_{i}(0)=\widetilde{v}_{i}\left(L_{z}\right)=0,
$$

which implies that the eigenfunctions [and, so, $U(z)$ and $\Delta(z)]$ can be expanded in the QWS given by

$$
\varphi_{l}(z)=\sqrt{2 / L_{z}} \sin \left[\pi(l+1) z / L_{z}\right] .
$$

Introducing the QWS components

$$
\widetilde{u}_{i}^{(l)}=\int d z \varphi_{l}^{*}(z) \widetilde{u}_{i}(z), \quad \widetilde{v}_{i}^{(l)}=\int d z \varphi_{l}^{*}(z) \widetilde{v}_{i}(z),
$$

we can rearrange the $\mathrm{BdG}$ equations as follows ( $i$ $\left.=\left\{k_{x}, k_{y}, j\right\}\right)$ :

$$
\begin{aligned}
E_{i} \widetilde{u}_{i}^{(l)}= & {\left[\frac{\hbar^{2}}{2 m}\left(\frac{\pi^{2}(l+1)^{2}}{L_{z}^{2}}+k_{x}^{2}+k_{y}^{2}\right)-\mu\right] \widetilde{u}_{i}^{(l)} } \\
& +\sum_{l^{\prime}}\left(U_{l l^{\prime}} \widetilde{u}_{i}^{\left(l^{\prime}\right)}+\Delta_{l l^{\prime}} \widetilde{v}_{i}^{\left(l^{\prime}\right)}\right), \\
E_{i} \widetilde{v}_{i}^{(l)}= & {\left[\mu-\frac{\hbar^{2}}{2 m}\left(\frac{\pi^{2}(l+1)^{2}}{L_{z}^{2}}+k_{x}^{2}+k_{y}^{2}\right)\right] \widetilde{v}_{i}^{(l)} } \\
& +\sum_{l^{\prime}}\left(\Delta_{l l^{\prime}} \widetilde{u}_{i}^{\left(l^{\prime}\right)}-U_{l l^{\prime}} \widetilde{v}_{i}^{\left(l^{\prime}\right)}\right)
\end{aligned}
$$

with

$$
\begin{aligned}
& \Delta_{l l^{\prime}}=\int d z \varphi_{l}^{*}(z) \Delta(z) \varphi_{l^{\prime}}(z), \\
& U_{l l^{\prime}}=\int d z \varphi_{l}^{*}(z) U(z) \varphi_{l^{\prime}}(z) .
\end{aligned}
$$

Note that $\Delta(z)$ and $U(z)$ are expressed in terms of $\widetilde{u}_{i}(z)$ and $\widetilde{v}_{i}(z)$ by means of Eqs. (3b) and (3a) where $u_{i}(\mathbf{r})$ and $v_{i}(\mathbf{r})$ are replaced by $\widetilde{u}_{i}(z)$ and $\widetilde{v}_{i}(z)$.

The coupled set of equations (11a) and (11b) were solved numerically by iterations until full self-consistency was reached. At the first iteration the bulk values for the anomalous and normal mean-field potentials $\Delta(z)=\Delta_{\text {bulk }}$ and $U(z)$ $=U_{\text {bulk }}$ were taken. At each iteration step the eigenvalues $E_{i}$ and corresponding eigenvectors $\left(\widetilde{u}_{i}^{(0)} \ldots \widetilde{u}_{i}^{(l)} \ldots \widetilde{v}_{i}^{(0)} \ldots \widetilde{v}_{i}^{(l)} \ldots\right)$ were calculated by transforming the governing matrix to a diagonal form and, then, new values of $U(z)$ and $\Delta(z)$ were found through Eqs. (3a) and (3b). In the case of strong deviations of the chemical potential from its bulk value (which is the case for very thin films with thickness less than 2 $-3 \mathrm{~nm}$ ), the iterative procedure was repeated for various $\mu$ until the mean electron density was obtained [see Eq. (5)]. The thickness-dependent superconducting temperature $T_{c}$ was found as the point above which $\Delta(z)=0$ is the only solution of Eqs. (11a) and (11b).

It is well known ${ }^{10}$ that the superconducting order parameter is not sensitive to the Hartree-Fock (normal) mean-field 
potential in case of a spatially homogeneous situation $\left(\Delta_{\text {bulk }}\right.$ remains the same for $U_{\text {bulk }}=0$ ). We confirmed, through a numerical investigation of the Bogoliubov-de Gennes equations, that a similar conclusion holds for nanofilms: though $\Delta(z)$ changes when including the normal mean-field potential, but this change is within a few percent. Therefore, in this work we present numerical solutions of Eqs. (11a) and (11b) for the case $U=0$.

\section{SHAPE RESONANCES IN THE SUPERCONDUCTING PROPERTIES}

The physics of the superconducting oscillations in the order parameter and in $T_{c}$ can be understood as follows. Since the classical papers by Gor' kov $^{11}$ and Bogoliubov ${ }^{12}$ it is known that the superconducting order parameter is the Cooper-pair wave function for the situation when both fermions have the same spatial coordinates. In other words, $\Delta(\mathbf{r})$ can be seen as the wave function describing the centerof-mass motion of a Cooper pair. The Cooper-pair wave function is influenced by the surrounding electrons of the Fermi sea ${ }^{13}$ so that the single-fermion states with energies well below the Fermi level do not make any essential contribution to the order parameter. As a result, $\Delta(\mathbf{r})$ will depend on the density of the single-fermion states (per unit volume and per spin projection) situated in the nearest vicinity of the Fermi level. Quantization of the electron motion in the direction normal to the film leads to the formation of QWS and a splitting of the band of single-electron states in a series of subbands. When the bottom of a given subband passes through the Fermi surface (with a change of film thickness), the density of single-electron states near the Fermi level increases abruptly. For nanofilms, this increase is significant. However, when approaching the bulk regime, the effect is washed out. Thus, the density of states per unit volume and per spin projection exhibits remarkable damped oscillations as a function of $L_{z}$. The same occurs for any superconducting property. It is important to note that the single-electron states located at the bottom of a subband give practically the same contribution to $\Delta(\mathbf{r})$ [it is determined by Eqs. (11a) and (11b) at $\left.k_{x}=k_{y}=0\right]$. This plays the role of amplifier: an increase of the superconducting quantities is more significant than a corresponding increase in the density of states. Thus, following Blatt and Thompson (see the first two articles in Ref. 8), the enhancement of superconductivity due to the passage of a QWS through the Fermi surface can be called a shape superconducting resonance.

The thickness-dependent superconducting temperature $T_{c}$ calculated self-consistently from Eqs. (11a) and (11b) is plotted in Fig. 1 in units of $T_{c \text {,bulk }}$ for four different metals (in our numerical calculations we took $L_{x}=L_{y}>500 \mathrm{~nm}$ to guarantee a sufficient accuracy, in order to be valid in the limit $L_{x}, L_{y}$ $\rightarrow+\infty$ ). The relevant parameters (see the textbooks ${ }^{9,10,14}$ ) are given in Table I. Here $k_{B}$ is the Boltzmann constant, $N_{\text {bulk }}(0)=m k_{F} /\left(2 \pi^{2} \hbar^{2}\right)$ denotes the bulk density of the single-electron states at the Fermi level and $k_{F}$ is the 3D Fermi wave number. Temperature dependence of the chemical potential is negligible for $T<T_{c}$ and, hence, we can write $k_{F}=\sqrt{2 m \mu_{\text {bulk }}} / \hbar$. As seen from Fig. 1, in general we obtain a

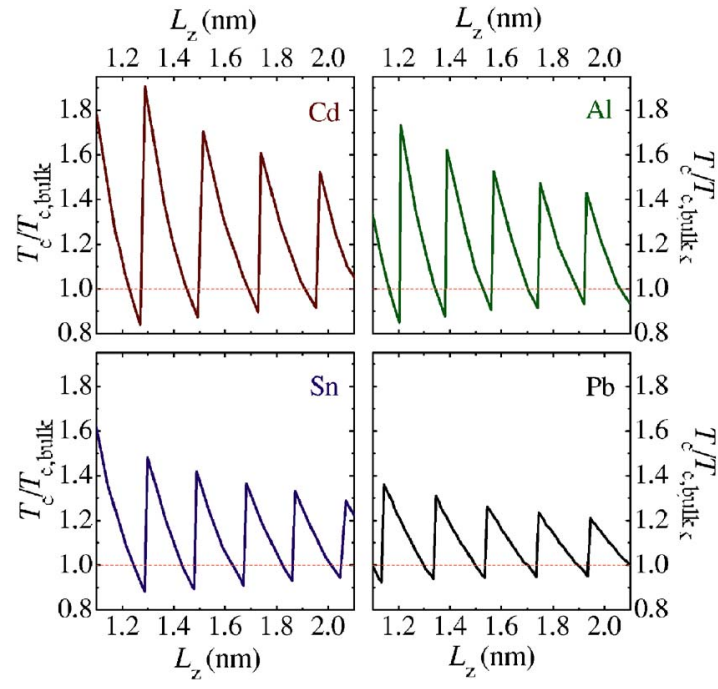

FIG. 1. (Color online) The relative critical temperature $T_{c} / T_{c \text {,bulk }}$ versus the film thickness $L_{z}$ for cadmium, aluminum, tin, and lead nanofilms.

sawtooth behavior for $T_{c}$ that is more complicated then the simple Friedel-like expression $\sim \cos \left(2 k_{F} L_{z}\right) / L_{z}^{\gamma}(\gamma \approx 1)$ typical for the thermal-stability function and the surface energy. ${ }^{2,3}$ At the resonant points the superconducting temperature increases well above its bulk value $T_{c \text {,bulk }}$. Whereas between two neighboring resonances $T_{c}$ moves down and even drops below $T_{c \text {,bulk. }}$. However, the resonance enhancements are more pronounced than depressions. Amplitudes of the shape resonances in $T_{c} / T_{c \text {,bulk }}$ are very sensitive to the governing parameters. Recall that the bulk superconducting quantities are determined by $\omega_{D}$ and $g N_{\text {bulk }}(0){ }^{9}{ }^{9} 10$ However, the resonant deviations depend on the three parameters $\omega_{D}$, $g$, and $\mu_{\text {bulk }}$ (the chemical potential $\mu$ appearing in the BdG equations depends on the mean electron density and, so, is fully determined by $\mu_{\text {bulk }}$ ). In other words, it is not sufficient to fix the product $g N_{\text {bulk }}(0)$ in this case. One should know the electron-phonon coupling $g$ and the bulk density of states $N_{\text {bulk }}(0)$ separately. Numerical investigation shows that oscillations of $T_{c} / T_{c}$,bulk are becoming less profound with increase of any of the parameters mentioned above. The point is that such increase results in an increase of the "condensation" energy of the superconducting electrons. In this situation some additional confinement energy is required to produce the same deviations from the bulk value, which results in a systematic shift of the shape superconducting resonances to smaller film thicknesses. It follows from Fig. 1 that cadmium ultrathin films exhibit the most profound resonances. In alu-

TABLE I. Parameters used in the numerical calculations.

\begin{tabular}{cccc}
\hline \hline Metal & $\hbar \omega_{D} / k_{B}$ & $g N_{\text {bulk }}(0)$ & Fermi level $\left(\mu_{\text {bulk }}\right)$ \\
\hline $\mathrm{Cd}$ & $164 \mathrm{~K}$ & 0.18 & $7.47 \mathrm{eV}$ \\
$\mathrm{Al}$ & $375 \mathrm{~K}$ & 0.18 & $11.7 \mathrm{eV}$ \\
$\mathrm{Sn}$ & $195 \mathrm{~K}$ & 0.25 & $10.2 \mathrm{eV}$ \\
$\mathrm{Pb}$ & $96 \mathrm{~K}$ & 0.39 & $9.47 \mathrm{eV}$ \\
\hline \hline
\end{tabular}




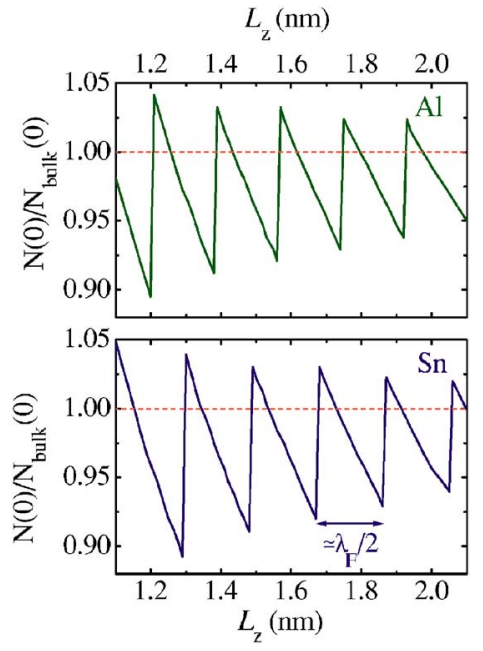

FIG. 2. (Color online) The density of single-electron states at the Fermi level $N(0)$ in units of the bulk density of states $N_{\text {bulk }}(0)$ as a function of the film thickness for aluminum and tin nanofilms.

minum they are less due to the larger Debye frequency and electron density. The shape superconducting resonances in tin and lead nanofilms are significantly suppressed by the stronger electron-phonon coupling.

It is instructive to compare the $T_{c}$ oscillations with the corresponding oscillations in the density of states at the Fermi level $N(0)$ [here $N(\xi)$ denotes the density of singleelectron states per unit volume and per spin projection at the energy $\xi$ measured from $\mu$ ]. Note that $N(0)$ was estimated as the number of relevant states (entering the Debye window) divided by $2 \hbar \omega_{D}$ and $V$. The estimation is accurate enough because $\hbar \omega_{D} \ll \mu$ in the case of interest. In Fig. 2 the thickness-dependent $N(0)$ extracted from a numerical solution of the BdG equations is plotted versus $L_{z}$ in units of $N_{\text {bulk }}(0)$ for $\mathrm{Al}$ and $\mathrm{Sn}$ films. The main feature of the $N(0)$ oscillations is that drops of $N(0)$ with respect to $N_{\text {bulk }}(0)$ are more pronounced than enhancements, and the amplitudes of these drops are not very sensitive to the material. The increase of $N(0) / N_{\text {bulk }}(0)$ at the resonant points is much less significant than the corresponding $T_{c}$ enhancement. The "amplification" factors (see the end of the first paragraph in this section) are about 6 and 4 for aluminum and tin, respectively. We remark that, in contrast to the naive expectation that $T_{c}$ should be equal to its bulk value when $N(0)=N_{\text {bulk }}(0)$, we find $T_{c}>T_{c \text {,bulk }}$ for such cases (see Fig. 1).

Numerical results demonstrate that the distance between two neighboring resonances is about $\pi / k_{F}$, which is one-half of the 3D Fermi wavelength $\lambda_{F}$ (see the Sn panel of Fig. 2). Single-electron states of a gas of noninteracting electrons are not exactly the same as such states in the presence of the Cooper pairing but they are close to each other. That is the reason why the above period of shape superconducting resonances can be explained in terms of the quantum well states $\varphi_{l}(z)$ given by Eq. (9). This $l$ state has the energy $\hbar^{2} \pi^{2}(l+1)^{2} /\left(2 m L_{z}^{2}\right)$ and, so, passes through the Fermi surface for film thickness $\hbar \pi(l+1) / \sqrt{2 m \mu}$. Hence, the distance between two neighboring resonances can be estimated as $\Delta L=\hbar \pi / \sqrt{2 m \mu}$. When $\mu$ approaches $\mu_{\text {bulk, }}$ we find $\Delta L$

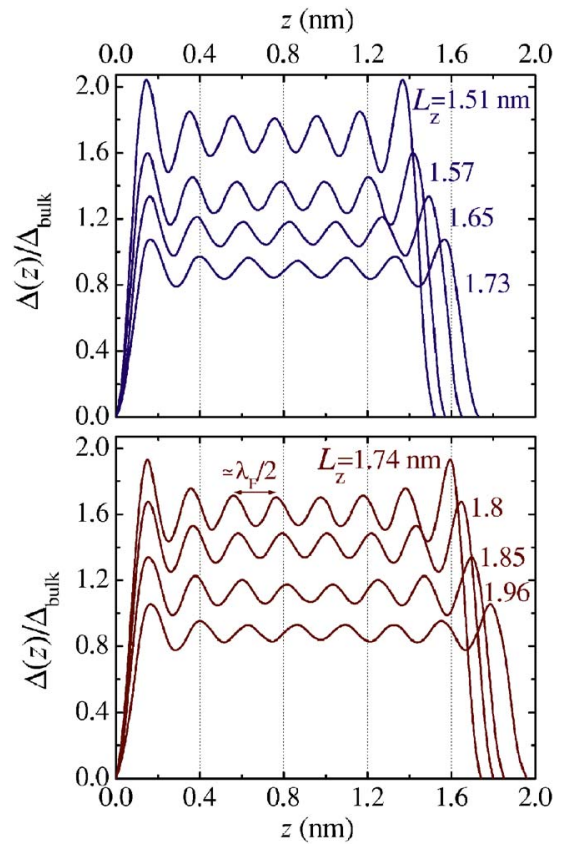

FIG. 3. (Color online) Spatial distribution of the relative superconducting order parameter $\Delta(z) / \Delta_{\text {bulk }}(z)$ in a Cd film of various thicknesses. The upper panel illustrates the decay of the shape superconducting resonance appearing at $L_{z}=1.51 \mathrm{~nm}$. The lower panel is for the resonance at $L_{z}=1.74 \mathrm{~nm}$.

$\simeq \lambda_{F} / 2$. To give a feeling about the thickness dependence of $\Delta L$, we remark that $\mu$ slowly increases with decreasing film thickness (this increase is not monotonic but shows little peaks at the resonant points). For instance, $\mu=11.2 \mathrm{eV}$ in a Sn film with thickness $L_{z}=1 \mathrm{~nm}$ while $\mu \rightarrow \mu_{\text {bulk }}=10.2 \mathrm{eV}$ near the bulk regime. Thus, in agreement with Fig. 2, $\Delta L$ $\simeq \lambda_{F} / 2$ within the accuracy of several percent provided that the film thickness $L_{z}>1 \mathrm{~nm}$.

QWS passages through the Fermi surface result not only in an increase of the superconducting quantities such as $T_{c}$ and $\Delta(z)$. They are also accompanied by a strongly nonuniform spatial distribution of the superconducting order parameter. In Fig. $3 \Delta(z)$ is plotted as a function of the $z$ coordinate normal to the film for the two resonant values of $L_{z}$ and beyond. Generally, $\Delta(z)$ is found to be an oscillating function with period that slightly decreases when moving from the sample boundaries to the center. For instance, when $L_{z}$ $=1.51 \mathrm{~nm}$, one-half of this period is about $0.146 \mathrm{~nm}$ near the boundaries but $0.105 \mathrm{~nm}$ in the center. At the resonant points $\left(L_{z}=1.51\right.$ and $1.74 \mathrm{~nm}$ in Fig. 3) the mean value of this period amounts to $\hbar \pi / \sqrt{2 m \mu}$, which is very close to $\lambda_{F} / 2$. While a shape resonance decays with increasing film thickness, the averaged period of the spatial oscillations in $\Delta(z)$ increases but the amplitude of such oscillations decrease together with the mean value of the superconducting order parameter. The number of peaks remains the same until the next shape resonance comes "into play." In the vicinity of this point a new peak arises in the center, and the averaged period of the spatial oscillations of the order parameter drops down to $\lambda_{F} / 2$. Such drops are determined by the ratio between the number of peaks in $\Delta(z)$ below and above the 
resonant points. If associating any shape superconducting resonance with such number $n$, the resonant point $L_{z}^{(n)}$ is then given by (when ignoring the thickness dependence of the Fermi level)

$$
2 k_{F} L_{z}^{(n)}=2 \pi n,
$$

which follows from our numerical results and is explained by the Bohr-Sommerfeld quantization rule. More accurate result for $L_{z}^{(n)}$ can be found when $k_{F}$ is replaced by $\sqrt{2 m \mu} / \hbar$ in Eq. (13). Concluding this section, we remind the reader that the Ginzburg-Landau (GL) theory was found to be very reliable when investigating mesoscopic superconductors (samples with micron and submicron dimensions). ${ }^{15}$ From Fig. 3 it follows that the GL approach becomes invalid for nanofilms where the superconducting order parameter is spatially inhomogeneous even in the absence of a magnetic field due to the shape superconducting resonances. Furthermore, $\Delta(z)=0$ at the edge of a nanofilm while the GL approach (supplemented by the conventional boundary condition) gives $\Delta(z) \neq 0$ at the edge of the film. Remember that the conventional boundary condition implies zero current through the sample surface. In the absence of a magnetic field the GL equations solved together with such a boundary condition result in a uniform spatial distribution of the Cooper pairs inside a superconducting specimen for any geometry (see, for instance, paragraph 5 in Sec. VI of Ref. 9).

\section{EFFECTIVE FERMI LEVEL}

As shown in the previous section, the distance between two neighboring superconducting resonances is close to $\lambda_{F} / 2$. Keeping to the standard bulk values for the Fermi level given in Table I, we find that $\lambda_{F} / 2$ is about $0.2 \mathrm{~nm}$ (provided that the parabolic band approximation is used). For instance, for $\mathrm{Pb}$, which is often used in experiments, this yields $\lambda_{F} / 2 \approx 0.7 a$, where $a=0.286 \mathrm{~nm}$ denotes the thickness of one monolayer (ML) in $\mathrm{Pb}(111) .{ }^{16}$ According to the above estimation, one could expect that a QWS passes through the Fermi surface in $\mathrm{Pb}(111)$ nanofilms with a period less than 1 ML. However, photoelectron spectroscopy demonstrates that this period is larger and close to $2 \mathrm{ML}^{4}$ To reproduce this value with the PB approximation we need to set $\mu_{\text {bulk }}$ $\approx 1 \mathrm{eV}$ which is almost an order of magnitude smaller than the value given in Table I. It is of importance to note that such disagreement was also found for other materials. Photoemission experiments on thin films of $\mathrm{Cu}, \mathrm{Ag}, \mathrm{Au}, \mathrm{Co}$, and Fe showed similar features. ${ }^{1}$ The general explanation is that the above problem is not connected with a depletion of the electron density in thin films but is an artifact of the PB approximation. ${ }^{1}$ One needs the actual band structure in the presence of quantum confinement to determine the period of the QWS passages through the Fermi surface in thin films. For instance, recent first-principle calculations of the quantized band structure of $\mathrm{Pb}(111)$ films have shown that the period in question is about $2.2 \mathrm{ML} .{ }^{16}$ Another theoretical result for this quantity is 1.8 ML. ${ }^{4}$ Both results are in agreement with the experimental observation of a period of $2 \mathrm{ML}^{4}$
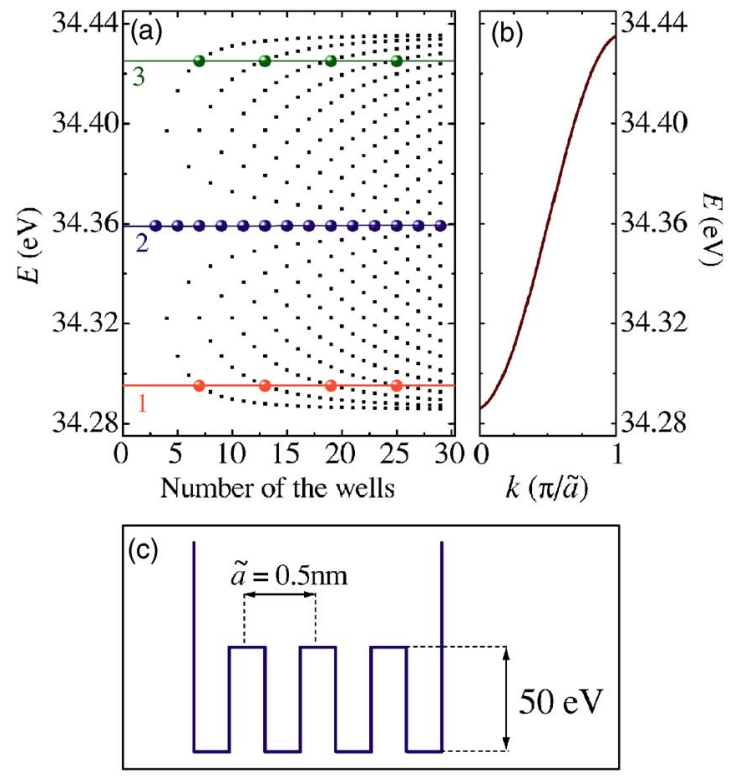

FIG. 4. (Color online) (a) The energy spectrum of a 1D electron placed in the external potential given schematically below in panel (c). The energies corresponding to the third Brillouin zone are plotted versus the number of wells. (b) is the the bulk energy dispersion for this Brillouin zone in the reduced zone scheme. Levels 1,2,3 are three possible positions of the Fermi level, and the thick solid circles represent the states passing through the Fermi surface.

To complement the discussion of the previous paragraph, we calculated the 1D energy spectrum of an electron in a model external potential given schematically in Fig. 4(c). In this simplified situation $\widetilde{a}$ is the analog of the lattice spacing and the number of wells situated between the infinite walls models the number of monolayers. In Fig. 4(a) the calculated energies of the QWS corresponding to the third Brillouin zone are shown versus the number of wells. The two states associated with the first and last wells (say, the surface states) are much higher in energy and not given here. The bulk energy dispersion for the third Brillouin zone is shown in the reduced zone representation in Fig. 4(b). To demonstrate how the periodicity of the QWS-passages through the Fermi surface depends on the Fermi level, three variants of its choice are highlighted here: level 1 is situated near the bottom of the zone, level 2 lies exactly in the center, and level 3 is near the edge of the zone. The relevant QWS are marked with the filled circles so that the period of the QWS passages is given by the distance between two neighboring circles. As seen, for level 1 the distance is $6 \widetilde{a}$. It is of importance to note that this result is also obtained within the PB approximation if we take $\mu_{\mathrm{bulk}}^{(1)}=10 \mathrm{meV}$ that is exactly the energy of level 1 measured from the bottom of the zone (but not $34.295 \mathrm{eV}$, measured from the bottom of the well). Note that the band mass is about $4.1 \mathrm{~m}$ in this illustrative example [it can be calculated from the dispersion in Fig. 4(b)]. The relevant formulas about the periodicity of the QWS passages (or shape resonances) in terms of the PB approximation can be found in the previous section. Level 3 shows the same QWS periodicity as level 1. It is interesting that to determine the electron band mass and the effective Fermi level for the PB approximation in this situation, the edge of the zone 


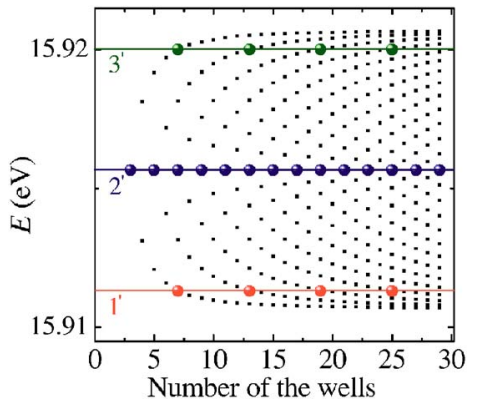

FIG. 5. (Color online) The same as in Fig. 4(a) but for the second Brillouin zone.

should be chosen as the reference point (see also the discussion in Ref. 4). The period of the QWS passages through the Fermi surface for level 2 is very close to the result of the PB approximation with an accuracy of about $10 \%$ even when introducing the effective Fermi level (of course, due to the location in the center of the zone). It does not matter whether the bottom or the edge of the third Brillouin zone is now taken as the reference point. Figure 5, where the energies of the QWS corresponding to the second Brillouin zone are plotted, demonstrates that the main aspects concerning the QWS periodicity do not change with the chosen zone. As seen, levels 1', 2', and 3' show the same period of the QWS passages as levels 1, 2, and 3 in Fig. 4. Also now the PB approximation gives good results if an effective Fermi level is used with the same features concerning the choice of the reference point.

Thus, based on the above simple picture, we expect that the PB approximation yields correct results for the period of the quantum-size oscillations when an effective Fermi level is introduced. It is important that in spite of the number of Brillouin zones being "in play," this effective Fermi level is determined by the relative position of the "true" Fermi level with respect to the bottom (or edge) of the highest occupied Brillouin zone in the plane corresponding to the film orientation. The BdG equations (11a) and (11b) are given in the PB approximation. Hence, though the qualitative tendencies and conclusions found in Sec. III are valid, in order to have relevant quantitative results we should incorporate an effective Fermi level rather than the values given in Table I. When such an effective Fermi level is used in the BdG equations, the product $g N_{\text {bulk }}(0)$ should be kept the same, in order to get the correct bulk limit for the superconducting order parameter and critical temperature.

\section{V. $T_{c}$ OSCILLATIONS FOR FILMS WITH AN EVEN (ODD) NUMBER OF MONOLAYERS}

In order to observe experimentally clear signatures of the QWS formation, one should grow ultrathin high quality films with atomic-scale uniformity in thickness. Therefore, the film thickness is expressed as an integer number of monolayers. In $\mathrm{Pb}(111)$ films QWS passes through the Fermi surface with a period of about $2 \mathrm{ML}$, which results in spectacular oscillations ${ }^{2-5}$ of the physical properties between films with even and odd number of monolayers (the even-odd or

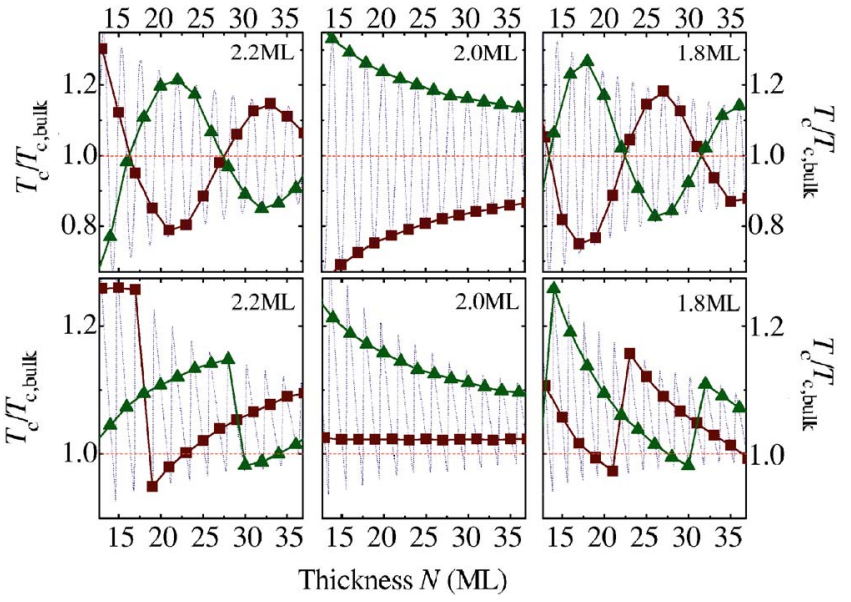

FIG. 6. (Color online) The thickness dependence of $T_{c} / T_{c \text {,bulk }}$ in odd-layered (squares) and even-layered (triangles) $\mathrm{Pb}(111)$ nanofilms for the QWS periods 2.2, 2.0, and 1.8 ML. The upper panels show results of the simplified Friedel-like approximation discussed in the text, the lower panels give the numerical results of the $\mathrm{BdG}$ equations.

bilayer oscillations). In addition, difference between $2 \mathrm{ML}$ and the real period of the QWS passages through the Fermi surface results in a beating effect on the bilayer oscillations. It implies relatively slow thickness-dependent oscillations of the physical properties in films made of even (or, alternatively, odd) number of monolayers, say, the even (odd) oscillations. These even (odd) oscillations lead to a switching of the film stability from even to odd number of monolayers and vice versa. ${ }^{4}$ In particular, when the thickness is smaller than $22 \mathrm{ML}$ only stable odd-layered $\mathrm{Pb}(111)$ films were obtained experimentally. ${ }^{4}$ Below we investigate the $T_{c}$ oscillations in the even- and odd-layered $\mathrm{Pb}(111)$ nanofilms.

In Fig. 6 the theoretical results for even (odd) oscillations of the superconducting temperature in $\mathrm{Pb}(111)$ nanofilms are given versus the number of monolayers $N$. The three upper panels show the data from a simplified Friedel-like approximation $T_{c} / T_{c \text {, bulk }}=A \cos \left(2 k_{F} a N\right) / N^{\gamma}+1$, where $\gamma=0.938$ (see Ref. 4) and, recall, $a=0.286 \mathrm{~nm}$ for $\mathrm{Pb}(111) .{ }^{16}$ The factor $A=13$ is chosen in such a way that amplitudes of the $T_{c}$ oscillations given in the upper panels are close to the amplitudes of the oscillations given in the lower panels, where $T_{c} / T_{c \text {,bulk }}$ is calculated using the BdG equations. The squares show the data for the odd-layered films whereas the triangles represent the even-layered films. The left panels correspond to the QWS-periodicity $2.2 \mathrm{ML}\left(\mu_{\text {bulk }}=1.07 \mathrm{eV}\right)$. In the central panels the distance between two neighboring shape superconducting resonances is about $2.0 \mathrm{ML}\left(\mu_{\text {bulk }}=1.25 \mathrm{eV}\right)$. The right panels are for the period 1.8 ML $\left(\mu_{\text {bulk }}\right.$ $=1.555 \mathrm{eV}$ ). Note that the results from the BdG equations are very different from the Friedel-like oscillatory behavior. For instance, the BdG results show a qualitative difference between the even (odd) oscillations in the left and right panels of the lower row in contrast to the simple Friedel-like approximation. The even (odd) oscillations disappear when the period of the QWS passages through the Fermi surface is exactly equal to $2 \mathrm{ML}$.

Figure 6 shows $T_{c}$ for so-called freestanding $\mathrm{Pb}(111)$ films. However, in experiments $\mathrm{Pb}$ films are grown on a $\mathrm{Si}(111)$ 


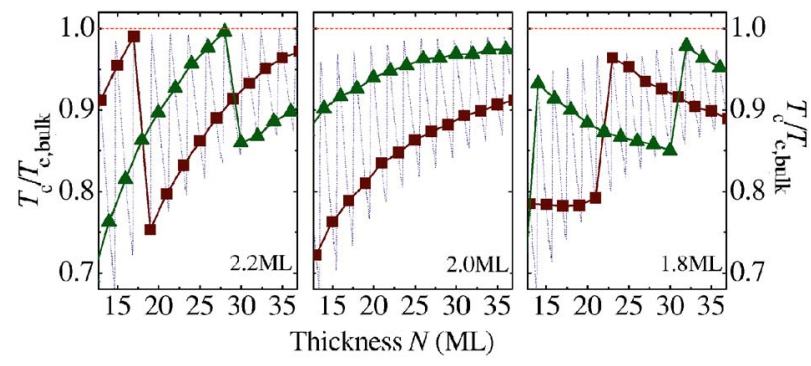

FIG. 7. (Color online) The relative superconducting temperature versus the number of the monolayers in odd-layered (squares) and even-layered (triangles) $\mathrm{Pb}(111)$ nanofilms for the QWS periods 2.2, 2.0, and 1.8 ML. The data have been calculated from the BdG equations with the thickness-dependent coupling constant $g$ given by Eq. (14) with $g_{1} N_{\text {bulk }}(0)=0.17$.

substrate, ${ }^{2-5,19}$ and the $T_{c}$ oscillations were reported for $\mathrm{Pb}$ (111) films with an Au coverage of about 4ML in thickness. Such substrate and coverage can change the electronphonon coupling constant in ultrathin films (with respect to the bulk) due to an interface effect. ${ }^{17}$ For instance, in ultrathin films (with $N<16 \mathrm{ML}$ ) made of $\mathrm{Ag}$ on a $\mathrm{Fe}(100)$ substrate the interface was shown to have a significant effect on the electron-phonon coupling which decreases down to its bulk value as the film thickness increases. ${ }^{17}$ Deviations of the coupling constant from the bulk limit follow approximately an overall $1 / N$ dependence. ${ }^{17}$ Note that the relative number of film atoms at the interface is proportional to $1 / N$. Hence, we may expect that the effect of the interface on the physical properties should on average scale as $\sim 1 / N$ (see discussion in Ref. 18), which is in agreement with the experimental result of Ref. 17. Measurements of the Eliashberg massenhancement factor $\lambda$ in $\mathrm{Pb}(111)$ ultrathin films ${ }^{4}$ demonstrate that in this case the electron-phonon coupling is lower than in bulk and gradually increases towards the bulk value with increasing film thickness. It is believed ${ }^{4}$ that such behavior is due to the influence of the silicon substrate. ${ }^{4}$ Hence, based on Refs. 4 and 17, it is reasonable to assume that, for the experimentally interesting situation of $\mathrm{Pb}(111)$ nanofilms grown on a silicon substrate, the electron-phonon coupling constant will depend on the film thickness. Following the above discussion, we approximate it by the form (for $N \geqslant 1$ )

$$
g=g_{0}-\frac{g_{1}}{N},
$$

where $g_{0} N_{\text {bulk }}(0)=0.39$ (this results in the correct bulk critical temperature and order parameter). The overall thicknessdependent drop of the superconducting temperature recently found in Refs. 4 and 19 for $\mathrm{Pb}(111)$ nanofilms with $N<30$ $\mathrm{ML}$ is reproduced when we take $g_{1} N_{\text {bulk }}(0) \sim 0.1-0.2$. Figure 7 illustrates how the $T_{c}$ oscillations are changed in the presence of the thickness-dependent coupling given by Eq. (14) (compare with Fig. 6 for the thickness independent coupling). Equations (11a) and (11b) were solved using Eq. (14) taking $g_{1} N_{\text {bulk }}(0)=0.17$ for the three different periods of the QWS passages through the Fermi surface 2.2, 2.0, and 1.8 ML. We found that the results corresponding to the period 1.8 ML are not in agreement with the general trend of the

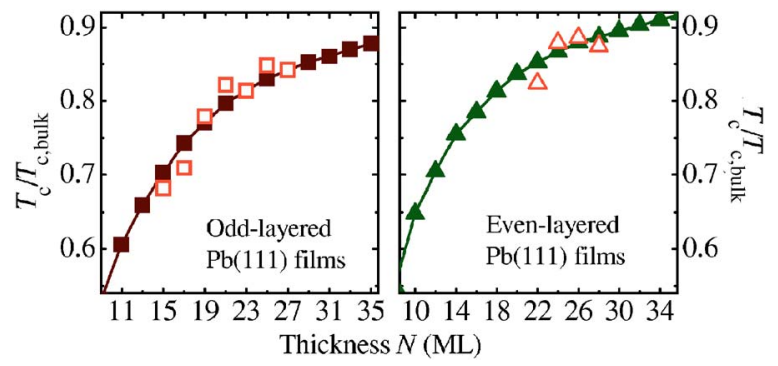

FIG. 8. (Color online) The superconducting temperature for the odd-layered (the left panel) and even-layered (the right panel) $\mathrm{Pb}(111)$ nanofilms: the empty squares and triangles represent the experimental data of Ref. 4; the filled squares (triangles) are the numerical results of the $\mathrm{BdG}$ equations with the thicknessdependent electron-phonon coupling constant given by Eq. (14) with $g_{1} N_{\text {bulk }}(0)=0.204\left[g_{1} N_{\text {bulk }}(0)=0.26\right]$.

experimental points (see Refs. 4 and 19). However, the experimental results are well reproduced if the QWS period is chosen to be about 2 ML or slightly larger than 2 ML. In Fig. 8 we present theoretical results for $T_{c}$ obtained when using the thickness-dependent electron-phonon coupling (filled squares and triangles for odd- and even-layered films, respectively) and compare them with the experimental data of Ref. 4 for $\mathrm{Pb}(111)$ nanofilms (empty squares for the oddlayered films and empty triangles for the even-layered films). The BdG equations were solved using $\mu_{\text {bulk }}=1.25 \mathrm{eV}$, which corresponds to a period of the QWS passages through the Fermi surface of about 2 ML. Good agreement with the experimental data for the odd-layered films is achieved when $g_{1} N_{\text {bulk }}(0)=0.204$ (see the left panel of Fig. 8). For the evenlayered $\mathrm{Pb}(111)$ films there are only few experimental points but we found that $g_{1} N_{\text {bulk }}(0) \approx 0.26$ in this case (see the right panel of Fig. 8). The difference in $g_{1}$ between the odd- and even-layered nanofilms is due to the fact that the approximation given by Eq. (14) provides only the correct baseline for the electron-phonon coupling constant but neglects the fine structure resulting in the even-odd oscillations. ${ }^{4,17}$ To get a more accurate approximation, a damped oscillatory term should be added to the expression in the right-hand side of Eq. (14). Then the electron-phonon coupling reads

$$
g=g_{0}-\frac{g_{*}+f\left(2 k_{F} a N\right)}{N},
$$

where $f(x)$ is a function oscillating around zero with period $2 \pi$ [compare Eq. (15) with the Friedel-like approximation given in the upper panels of Fig. 6]. Now variations of the electron-phonon coupling follow a damped oscillatory curve superimposed on the base line $g_{0}-g_{*} / N$. When the period of the QWS passages through the Fermi surface is given by $\pi / k_{F}=2 a$ (or $2 \mathrm{ML}$ ), from Eq. (15) we find $g=g_{0}$ $-\left[g_{*}+f(\pi)\right] / N$ for the odd-layered films and $g=g_{0}$ $-\left[g_{*}+f(2 \pi)\right] / N$ for the even-layered films. This explains the above difference in $g_{1}$. Putting, for the sake of simplicity, $f\left(2 k_{F} a N\right)=\cos \left(2 k_{F} a N\right)$ (hence, invoking a Friedel-like approximation for the electron-phonon coupling), we get $g_{*}$ $=0.232 / N_{\text {bulk }}(0)$ and $f(2 \pi)=-f(\pi)=0.028 / N_{\text {bulk }}(0)$ for the 


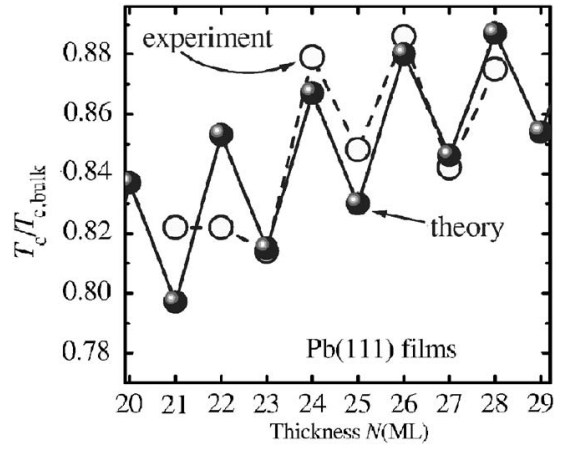

FIG. 9. The even-odd oscillations in the critical temperature of $\mathrm{Pb}$ (111) films on silicon: empty circles represent the experimental data from Ref. 4, solid circles are numerical results of the BdG equations (parameters are the same as in the previous figure).

theoretical data given in Fig. 8. Our numerical results reproduce not only the experimental trends of $T_{c}$ in the odd- and even-layered $\mathrm{Pb}(111)$ nanofilms but also the fine structure of the even-odd oscillations (see Fig. 9). Note that in the experiments of Ref. 4 the even-layered $\mathrm{Pb}(111)$ nanofilms were found to be rough and unstable for $N<22 \mathrm{ML}$. This can be a solid reason for a difference between our predictions and experimental results at $N=22 \mathrm{ML}$. One can expect that being at the edge of the stability region, a $\mathrm{Pb}(111)$ flat terrace with $N=22 \mathrm{ML}$ was not as perfect as the even-layered terraces with $N>22$ ML. Fluctuations in film thickness can significantly smooth and even wash out the quantum oscillations of the superconducting temperature (see the papers by Blatt and Thompson in Ref. 8). In addition, structural defects ${ }^{19}$ can also be responsible for the difference between the theoretical and experimental data in Fig. 9.

The critical temperature in $\mathrm{Pb}$ (111) films with an $\mathrm{Au}$ cap cover $^{4}$ and with an amorphous Ge cap layer ${ }^{19}$ were found to be different. We may interpret this as due to different interface effects, which leads to small changes in the electronphonon coupling constant, i.e., $g_{1}$ in Eq. (14). In Fig. 10 we show that our calculations are in agreement with the results of Ref. 19 when $g_{1} N_{\text {bulk }}(0)=0.077$ for the odd-layered nanofilms and $g_{1} N_{\text {bulk }}(0)=0.177$ for the even-layered specimens. In terms of Eq. (15) this gives $g_{*}=0.127 / N_{\text {bulk }}(0)$ and $f(2 \pi)=-f(\pi)=0.05 / N_{\text {bulk }}(0)$. From Figs. $8-10$ we find that there is little difference in $T_{c}$ for odd- and even-layered $\mathrm{Pb}(111)$ nanofilms (compare with Figs. 6 and 7). The even-

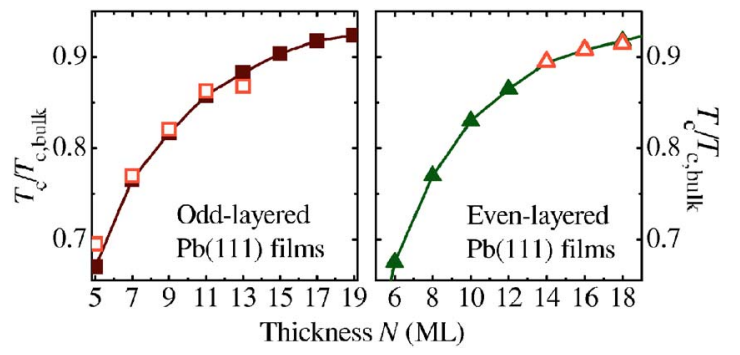

FIG. 10. (Color online) The same as Fig. 8 but the experimental data are now from Ref. 19 and theoretical results correspond to $g_{1} N_{\text {bulk }}(0)=0.077$ for the odd-layered nanofilms and $g_{1} N_{\text {bulk }}(0)$ $=0.177$ for the even-layered films. odd oscillations in the critical temperature are significantly weakened by the presence of the even-odd oscillations in the thickness dependence of the electron-phonon coupling constant. Another important consequence of Eqs. (14) and (15) is a gradual decrease in the superconducting temperature with reducing film thickness. This is in marked contrast with recent results ${ }^{20}$ for $\mathrm{Al}$ nanowires where $T_{c}$ was found to increase with decreasing thickness. However, we should stress that a silicon substrate influences the results for $\mathrm{Pb}(111)$ nanofilms with thickness less than $30 \mathrm{ML}$ (about $9 \mathrm{~nm}$ ). For thicker films the effect is washed out and the electronphonon coupling approaches the bulk value. In this situation we arrive at the regime illustrated in the lower panels of Fig. 6. As seen, this regime is characterized by an overall increase of the thickness-dependent $T_{c}$ when the film thickness decreases. Such increase was recently observed in Al nanowires, fabricated on a silicon substrate, with effective diameter $\geqslant 10 \mathrm{~nm}^{20}$

We want to stress that an oversimplified explanation of the $T_{c}$ drop in ultrathin $\mathrm{Pb}(111)$ nanofilms invoking the GL theory with an extra surface term ${ }^{21}$ is not correct. Such an explanation as that given in Ref. 19 ignores the fact that the GL theory with the surface term predicts not only a drop of the critical temperature in ultrathin nanofilms but also a decrease of the critical perpendicular and parallel magnetic fields while the experimental data ${ }^{5}$ show an increase of the perpendicular upper critical magnetic field in $\mathrm{Pb}(111)$ nanofilms.

We remark that atomically flat $\mathrm{Pb}$ terraces with width of about 1-2 $\mu \mathrm{m}$ were investigated in Refs. 4 and 19. Very recently the data for $T_{c}$ in flat $\mathrm{Pb}$ islands grown on $\mathrm{Si}(111)$ surface were reported (their width is about $50-100 \mathrm{~nm}$ ). ${ }^{22,23}$ It is interesting that the superconducting critical temperature was found to be rather sensitive to the island width ${ }^{23}$ and higher than $T_{c}$ in $\mathrm{Pb}$ terraces. In order to go in more detail about this problem, several points should be kept in mind. First, contrary to the experiments of Refs. 4 and 19, lead islands investigated in the papers ${ }^{22,23}$ were produced without protecting cover caps. Second, scanning tunnelling spectroscopy used in Refs. 22 and 23 is a local probe. On the contrary, the results for $T_{c}$ in the $\mathrm{Pb}$ terraces were extracted from measurements of the temperature dependent resistance and upper critical magnetic field that involve an averaging over the whole sample. ${ }^{4,19}$ At last, quantum growth defects ${ }^{19}$ can contribute to a quenching of the electron-phonon coupling found in the experiments with lead terraces on silicon. ${ }^{4}$

\section{CONCLUSION}

Quantum-size variations of $T_{c}$ in atomically uniform metallic nanofilms with thickness less than the mean free electron path were studied through a self-consistent numerical solution of the Bogoliubov-de Gennes equations. We found pronounced oscillations in $T_{c}$ with decreasing film thickness. The maxima of these oscillations are substantially above $T_{c \text {,bulk }}$ while the minima are just below (about 10\%) $T_{c \text {,bulk. }}$. The resonant enhancements of $T_{c}$ are largest for $\mathrm{Cd}$ and smallest in $\mathrm{Pb}$ nanofilms. Renormalization of the electronphonon coupling due to the presence of a substrate (and a 
protecting cover, as well) may reduce the predicted enhancements in $T_{c}$. When comparing with recent experimental results on $\mathrm{Pb}(111)$ flat terraces grown on silicon, we found that the damped oscillatory decrease in the electron-phonon coupling constant strongly reduces the superconducting temperature and smoothen its quantum-size oscillations. Based on related experiments on the width dependence of nanofilm properties (see Refs. 2, 3, and 17) we assumed a simple damped Friedel-like analytical expression for the electronphonon coupling constant with two fitting parameters. Insert- ing this expression into our numerical solution for the Bogoliubov-de Gennes equations, we found excellent agreement with the recent experimental results on the width dependence of $T_{c}$ in $\mathrm{Pb}(111)$ nanofilms.

\section{ACKNOWLEDGMENTS}

This work was supported by the Flemish Science Foundation (FWO-Vl), the Belgian Science Policy, and BOF-TOP (University of Antwerp).
${ }^{1}$ J. E. Ortega and F. J. Himpsel, Phys. Rev. Lett. 69, 844 (1992); N. V. Smith, N. B. Brookes, Y. Chang, and P. D. Johnson, Phys. Rev. B 49, 332 (1994); E. Hüger and K. Osuch, J. Electron Spectrosc. Relat. Phenom. 141, 13 (2004).

${ }^{2}$ P. Czoschke, Hawoong Hong, L. Basile, and T.-C. Chiang, Phys. Rev. Lett. 93, 036103 (2004).

${ }^{3}$ M. H. Upton, C. M. Wei, M. Y. Chou, T. Miller, and T.-C. Chiang, Phys. Rev. Lett. 93, 026802 (2004).

${ }^{4}$ Yang Guo, Yan-Feng Zhang, Xin-Yu Bao, Tie-Zhu Han, Zhe Tang, Li-Xin Zhang, Wen-Guang Zhu, E. G. Wang, Qian Niu, Z. Q. Qiu, Jin-Feng Jia, Zhong-Xian Zhao, and Qi-Kun Xue, Science 306, 1915 (2004); Yan-Feng Zhang, Jin-Feng Jia, Tie-Zhu Han, Zhe Tang, Quan-Tong Shen, Yang Guo, Z. Q. Qiu, and Qi-Kun Xue, Phys. Rev. Lett. 95, 096802 (2005).

${ }^{5}$ Xin-Yu Bao, Yan-Feng Zhang, Yupeng Wang, Jin-Feng Jia, QiKun Xue, X. C. Xie, and Zhong-Xian Zhao, Phys. Rev. Lett. 95, 247005 (2005).

${ }^{6}$ B. G. Orr, H. M. Jaeger, and A. M. Goldman, Phys. Rev. Lett. 53, 2046 (1984).

${ }^{7}$ O. Pfennigstorf, A. Petkova, H. L. Guenter, and M. Henzler, Phys. Rev. B 65, 045412 (2002).

${ }^{8}$ J. M. Blatt and C. J. Thompson, Phys. Rev. Lett. 10, 332 (1963); C. J. Thompson and J. M. Blatt, Phys. Lett. 5, 6 (1963); E. H. Hwang, S. Das Sarma, M. A. Stroscio, Phys. Rev. B 61, 8659 (2000).

${ }^{9}$ P. G. de Gennes, Superconductivity of Metals and Alloys (W. A. Benjamin, New York, 1966).
${ }^{10}$ A. L. Fetter and J. D. Walecka, Quantum Theory of ManyParticle Systems (Dover, New York, 2003).

${ }^{11}$ L. P. Gor'kov, Sov. Phys. JETP 7, 505 (1958).

${ }^{12}$ N. N. Bogoliubov, Sov. Phys. Usp. 67, 549 (1959).

${ }^{13}$ L. N. Cooper, Phys. Rev. 104, 1189 (1956).

${ }^{14}$ N. W. Ashcroft and N. D. Mermin, Solid State Physics (Saunders, New York, 1976).

${ }^{15}$ V. V. Moshchalkov, L. Gielen, C. Strunk, R. Jonckheere, X. Qiu, C. Van Haesendonck, and Y. Bruynseraede, Nature (London) 373, 319 (1995); A. K. Geim, I. V. Grigorieva, S. V. Dubonos, J. G. S. Lok, J. C. Maan, A. E. Filippov, and F. M. Peeters, ibid. 390, 259 (1997); P. S. Deo, V. A. Schweigert, F. M. Peeters, and A. K. Geim, Phys. Rev. Lett. 79, 4653 (1997); V. A. Schweigert, F. M. Peeters, and P. S. Deo, ibid. 81, 2783 (1998).

${ }^{16}$ C. M. Wei and M. Y. Chou, Phys. Rev. B 66, 233408 (2002).

${ }^{17}$ D. A. Luh, T. Miller, J. J. Paggel, and T.-C. Chiang, Phys. Rev. Lett. 88, 256802 (2002).

${ }^{18}$ Tai-Chang Chiang, Science 306, 1900 (2004).

${ }^{19}$ M. M. Özer, J. R. Thompson, and H. H. Weitering, Nat. Phys. 2, 173 (2006).

${ }^{20}$ A. A. Shanenko, M. D. Croitoru, M. Zgirski, F. M. Peeters, and K. Arutyunov, Phys. Rev. B 74, 052502 (2006).

${ }^{21}$ J. Simonin, Phys. Rev. B 33, 7830 (1986).

${ }^{22}$ D. Eom, S. Qin, M.-Y. Chou, and C. K. Shih, Phys. Rev. Lett. 96, 027005 (2006).

${ }^{23}$ T. Nishio, M. Ono, T. Eguchi, H. Sakata, and Y. Hasegawa, Appl. Phys. Lett. 88, 113115 (2006). 